\title{
Note clinique : Un cas de globidiose cutanée bovine
}

\author{
par A. PROVOST (*)
}

\begin{abstract}
RESUME
L'auteur rapporte un cas de globidiose cutanée bovine observé dans lo nord du Cameroun.
\end{abstract}

La globidiose cutanée bovine est connue en pathologie tropicale mais les cas décrits sont rares. HENNING (2) en a fait une bonne description, incluant une revue bibliographique exhaustive. Plus récemment, BWANGAMOI (1) rapporte deux cas nécropsiques chez des chèvres du Kenya dont les peaux présentaient des lésions de dimple.

Sans doute est-ce l'absence de figures dans les manuels de pathologie ou de protozoologie vétérinaire qui fait que les cliniciens n'ont pas l'esprit frappé par l'aspect, pourtant caractéristique, de la peau des malades en voie de guérison. A notre connaissance, il n'existe que peu de photographies de qualité de cette condition: celles du livre de HENNING (2) sont médiocres, celles du traité de SMITH et JONES (4) un peu meilleures; seules les planches de la publication de POLS (3) sont plus parlantes. Nous pensons faire cuvre utile en présentant les photographies ci-jointes, à verser au dossier clinique de la globidiose cutanée.

\section{Présentation du cas}

Le 21 février 1965, expérimentant en brousse un vaccin antipéripneumonique dans la région de Garoua (Cameroun), notre attention fut attirée par l'aspect inusité du poil d'une vache d'un troupeau passant sur la route.

(") I.E.M.V.T., Laboratoire de Recherches de Farcha, B.P. 433, N'Djamena, Tchad.
Le bouvier se prêtait volontiers à l'examen de son animal sur lequel purent être photographiées les lésions et réalisé un grattage de peau jusqu'à la rosée sanguine.

La peau des épaules, du dos et des fesses avait un aspect cartonné, épaissi, présentant de nombreuses arêtes en relief dessinant une sorte de quadrillage grossier; le sommet des arêtes était alopécique. Au niveau des crêtes iliaques, elle était totalement glabre, hyperkératosique, sèche, sans suintement. Aucune lésion de grattage n'était visible. L'animal ne paraissait pas souffrir outre mesure de sa condition.

De retour au laboratoire, de nombreux kystes de Besnoitia besnoiti étaient visibles au microscope dans le raclage de la peau. L'animal était le seul dans un troupeau d'une vingtaine de têtes à présenter ces lésions cutanées. L'interrogatoire du bouvier n'a permis d'obtenir aucun renseignement valable, si ce n'est que les animaux n'avaient reçu aucun trypanocide à action éventuellement photosensibilisante.

A quelques jours de là, un autre cas de globidiose bovine, à forme mixte intestinale et cutanée, a été observé sur une génisse près de Massakory, au Tchad. L'animal présentait une diarrhée très fluide, hémorragique, avec un début d'hyperkératose de la face postérieure des cuisses. $B$. besnoiti était facilement mis en évidence. 


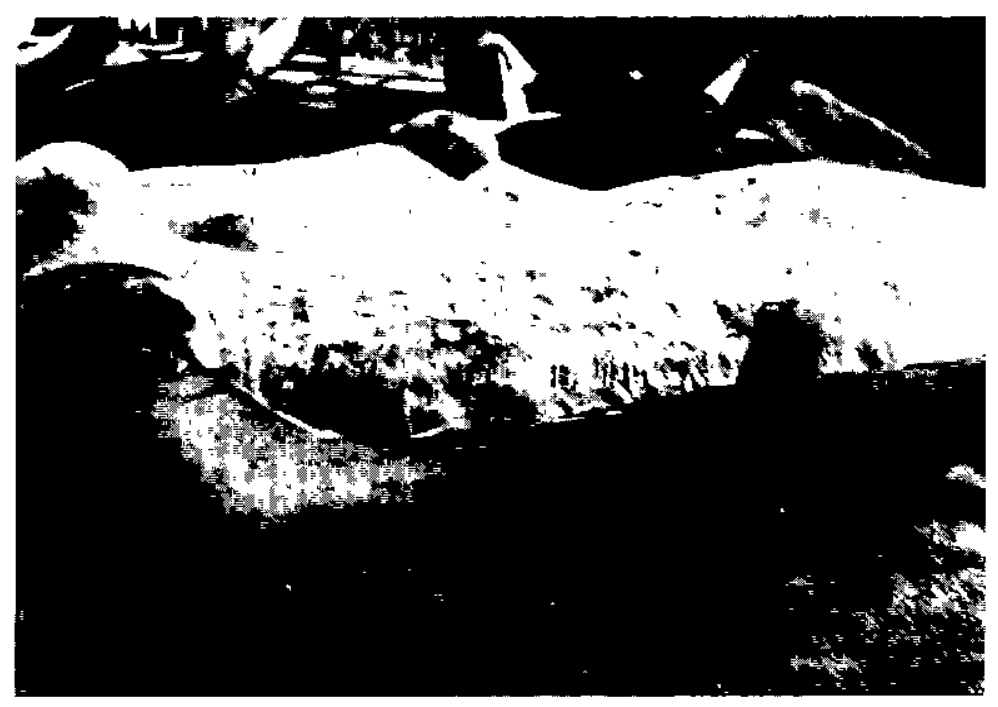

Photo 1. - Répartition des lésions cutanées sur les épaules, le dos et les fesses.

Photos 2 et 3. - Aspect hyperkeratosique de la peau des fesses.
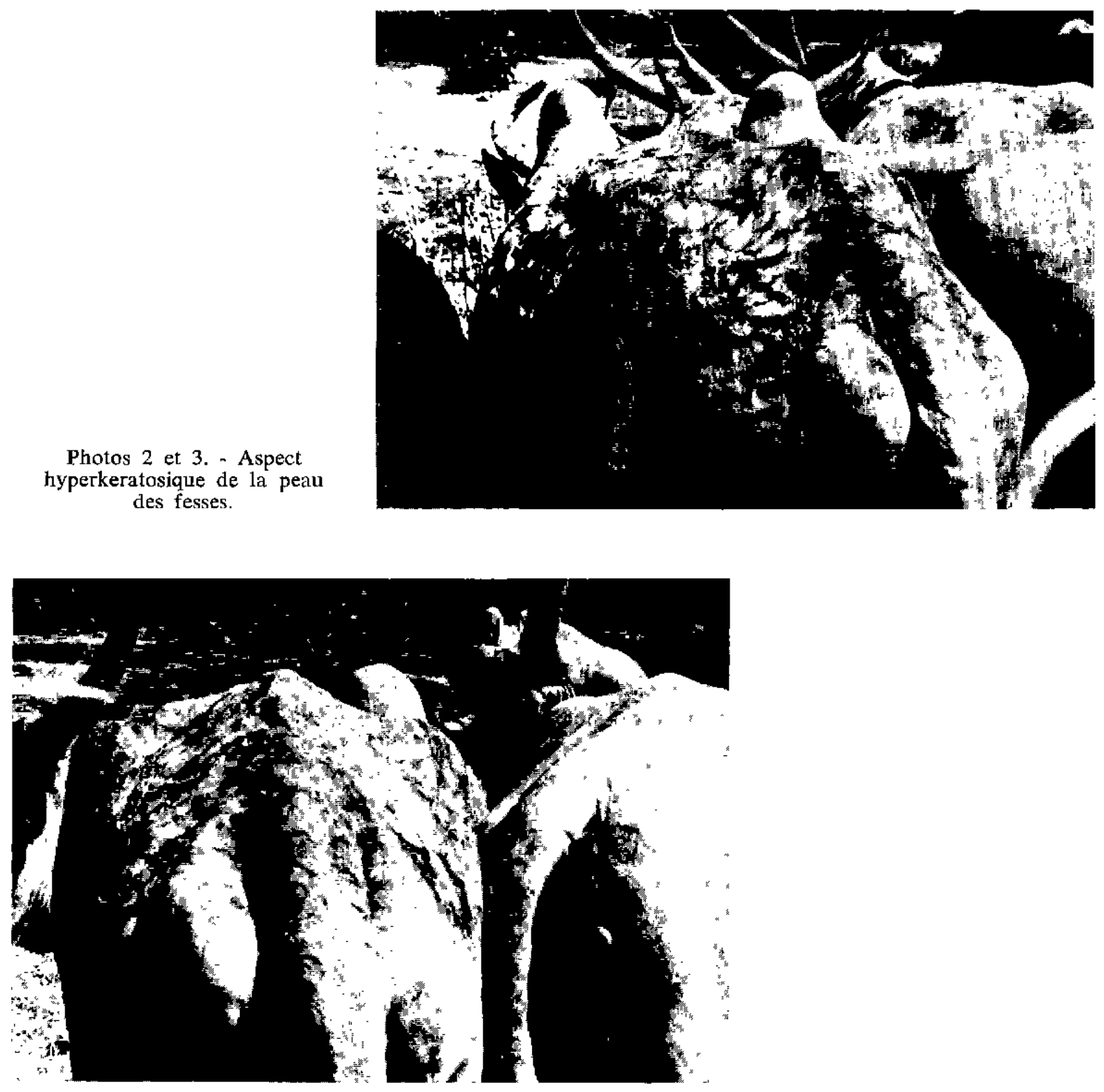
Cette note sans prétention ne vise qu'à illustrer la clinique de cette curieuse maladie bovine et attirer l'attention des cliniciens sur la forme dite de séborrhée sèche, phase terminale de la maladie.

\section{Remerciements}

L'auteur est heureux de remercier le personnel du Service de l'Elevage de la République Unie du Cameroun (secteur Nord d'Elevage) avec qui le, cas a été observé.

\section{SUMMARY}

A case of cutaneous bovine glcbidiosis observed in northern Cameroon is reported by the author.

\section{RESUMEN}

Nota clinica :

un caso de globidiosis cutánea bovina

El autor nota un caso de globidiosis cutánea bovina observado en el norte de Camerún.

\section{BIBLIOGRAPHIE}

1. BWANGAMOI (O.). A preliminary report on the finding of Besnoitia besnoiti in goat skins affected with dimple in Kenya. Bull. epiz. Dis. Afr., 1967, 15: $263-271$.

2. HENNING (M.W.). Animal Diseases in South Africa. South Africa, Central News Agency Ltd., 1956.
3. POLS (J. W.). Studies on bovine besnoitiosis with special reference to the aetiology. Onderstepoort J. vet. Res., 1960, 28 : 265-356.

4. SMITH (H. A.) et JONES (T. C.). Veterinary Pathology. Philadelphia, Lea and Febriger, 1961. 